\title{
Contrast-Enhanced Endoscopic Ultrasound for Evaluation of Common Bile Duct in Presence of Portal Cavernoma
}

\author{
Surinder Singh Rana ${ }^{1}$ Ravi Kumar Sharma Rajesh Gupta² \\ ${ }^{1}$ Department of Gastroenterology, Postgraduate Institute of \\ Medical Education and Research (PGIMER), Chandigarh, India \\ 2Department of Surgical Gastroenterology, Postgraduate Institute \\ of Medical Education and Research (PGIMER), Chandigarh, India \\ Address for correspondence Surinder S Rana, MD, DM, \\ Department of Gastroenterology, Postgraduate Institute of Medical \\ Education and Research (PGIMER), Chandigarh 160012, India \\ (e-mail: drsurinderrana@gmail.com).
}

\begin{abstract}
Keywords

- endosonography

- common bile duct

- gall bladder stones

Endoscopic ultrasound (EUS) is an excellent imaging modality for the evaluation of common bile duct (CBD) because of its close proximity to the transducer placed in duodenum. However, in the presence of portal cavernoma, identification and proper evaluation of CBD become difficult because of presence of numerous venous collaterals. In these circumstances, the evaluation of CBD is more difficult if it is nondilated. In these difficult situations, contrast-enhanced EUS can provide better and clear images of CBD and therefore seems to be an excellent modality to evaluate nondilated CBD in the presence of portal cavernoma.
\end{abstract}

\section{Case Report}

A 32-year-old man, known case of extrahepatic portal venous obstruction, presented with upper abdominal pain. Ultrasound abdomen revealed multiple gall bladder stones with portal cavernoma and common bile duct (CBD) could not be evaluated. Serum bilirubin as well as alanine and aspartate transaminase levels were normal but alkaline phosphatase (ALP) was elevated (368 IU/L; N,126 IU/L). Magnetic resonance imaging could not be done because of patient's claustrophobia. Endoscopic ultrasound (EUS) was performed using radial echoendoscope (GF-UE160-AL5; Olympus Corp., Tokyo, Japan) and universal ultrasound processor EU-ME2 premier plus (Olympus Corp., Tokyo, Japan). EUS performed from duodenal bulb revealed multiple serpiginous linear anechoic structures in suprapancreatic region and CBD could not be separately seen ( $\boldsymbol{- \text { Fig. }} \mathbf{1}$ ). On Doppler EUS, all these serpiginous structures showed flow and in center of these vascular collaterals small, round, nonvascular, anechoic CBD ( - Fig. 2; arrows) could be seen. However, because of multiple venous collaterals, CBD could not be properly visualized. Thereafter, contrast-enhanced harmonic EUS (CH-EUS) using
Sonovue (Bracco, Milan, Italy) was done at low mechanical index of 0.12 . Contrast-highlighted venous collaterals $50 \mathrm{sec}-$ onds after injection of contrast and nonenhancing CBD could be completely and clearly visualized ( - Figs. $\mathbf{3} \mathbf{A}$ and $\mathbf{B}$ arrows and -Video 1). The contrast-filled echogenic para-choledochal venous collaterals were clearly visualized separate from anechoic, noncontrast-filled CBD. The CBD was found to be normal and patient had low serum vitamin $D$ levels. Serum ALP normalized after correction of vitamin D levels and patient was referred to surgical services for cholecystectomy.

\section{Take-Home Message}

EUS is an excellent imaging modality for the evaluation of $\mathrm{CBD}$ because of its close proximity to the transducer placed in duodenum. ${ }^{1}$ In the presence of portal cavernoma, identification and evaluation of CBD become difficult because of presence of numerous venous collaterals. ${ }^{1,2}$ In these circumstances, the evaluation of CBD is more difficult if it is nondilated. ${ }^{2,3} \mathrm{CH}$-EUS seems to be an excellent modality to evaluate nondilated CBD in the presence of portal cavernoma. 

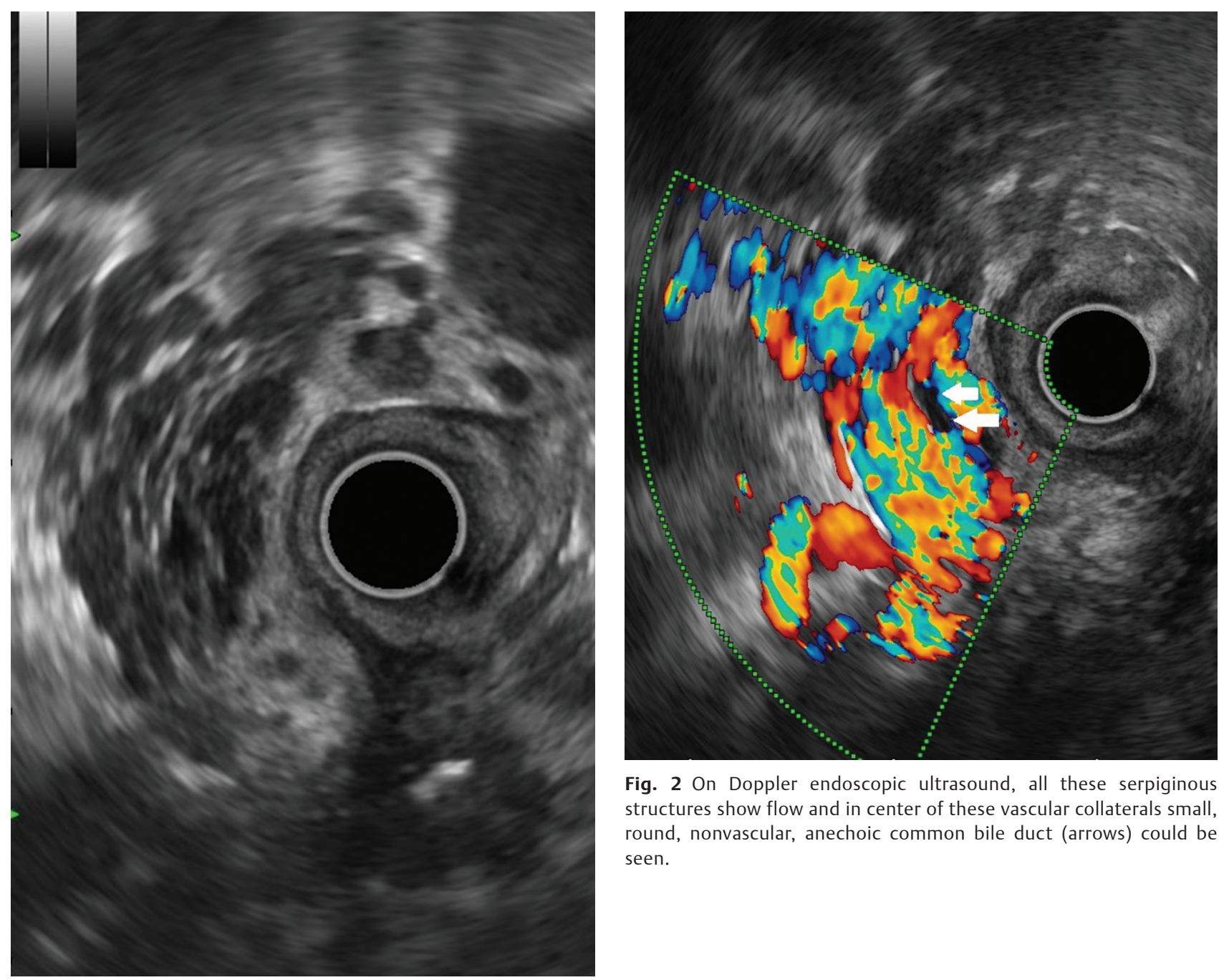

Fig. 2 On Doppler endoscopic ultrasound, all these serpiginous structures show flow and in center of these vascular collaterals small, round, nonvascular, anechoic common bile duct (arrows) could be seen.

Fig. 1 Endoscopic ultrasound from duodenal station: multiple serpiginous linear anechoic structures in suprapancreatic region and common bile duct could not be separately seen
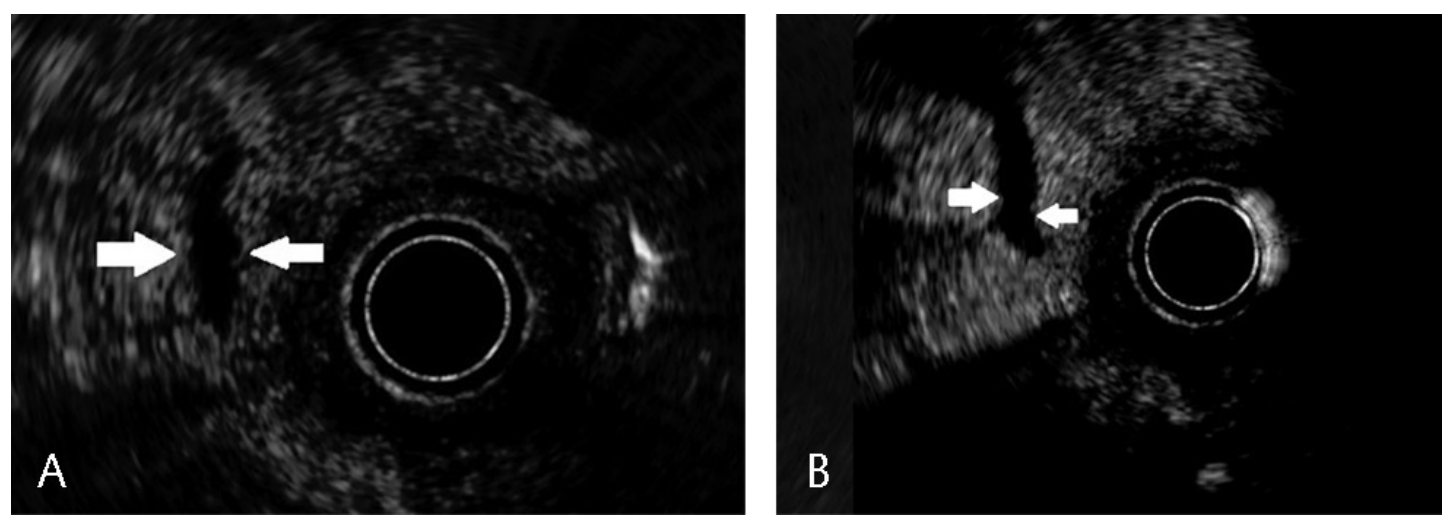

Fig. 3 (A and B) Contrast-enhanced harmonic EUS: The contrast-filled echogenic paracholedochal venous collaterals clearly visualized separate from anechoic, noncontrast-filled common bile duct. 


\section{Video 1}

Noncontrast endoscopic ultrasound shows multiple serpiginous linear anechoic structures in suprapancreatic region and common bile duct (CBD) could not be separately seen. After injection of intravenous contrast, the echogenic paracholedochal venous collaterals clearly visualized separate from anechoic, noncontrast-filled CBD. The CBD could be clearly seen throughout its extent. Online content including video sequences viewable at: https://www.thieme-connect.com/products/ejournals/ html/doi/10.1055/s-0040-1716790.

\section{Author Contributions}

1. Surinder Singh Rana: Collection and interpretation of data, drafting of manuscript.

2. Ravi Sharma: Collection and interpretation of data.

3. Rajesh Gupta: Collection and interpretation of data.

\section{Financial Disclosures}

No financial disclosures.

Informed consent was obtained from the patient for the publication of their information and imaging.

\section{Conflicts of Interest}

None.

\section{References}

1 Palazzo L, Hochain P, Helmer C, et al. Biliary varices on endoscopic ultrasonography: clinical presentation and outcome. Endoscopy 2000;32(7):520-524

2 Rana SS, Bhasin DK, Behera A. Rare cause of jaundice in chronic pancreatitis: portal hypertensive biliopathy. Clin Gastroenterol Hepatol 2011;9(7):e64-e65

3 Rana SS, Bhasin DK, Rao C, Singh K. Portal hypertensive biliopathy developing after acute severe pancreatitis. Endosc Ultrasound 2013;2(4):228-229 